\title{
Non-pharmacological interventions for persons with dementia: what are they and how should they be studied?
}

The publication of four papers concerning nonpharmacological interventions for persons with dementia heralds progress in the science of dementia care. The four papers are very diverse in focus and methodology, and include a study of the impact of a visual arts program on quality of life, communication, and well-being by Windle et al. (2017); an overview of systematic reviews of pharmacological and non-pharmacological interventions for the treatment of behavioral and psychological symptoms of dementia by Dyer et al. (2017); a systematic review of the efficacy of intervention in people with Lewy body dementia by Morrin et al. (2017); and a protocol of the Behavior and Evolution of Young Onset Dementia part two (BEYOND-II) study, an intervention study aimed at improvement in the management of neuropsychiatric symptoms in institutionalized people with young onset dementia by van Duinenvan den IJssel et al. (2017).

The publication of this combination of wideranging papers on non-pharmacological interventions for persons with dementia raises the opportunity to discuss basic questions concerning such research. These questions pertain to the definition and understanding of non-pharmacological interventions, understanding the role of nonpharmacological interventions within subgroups of different types of dementia, and appropriate methodologies for examining the impact of nonpharmacological intervention on persons with dementia.

\section{What is a non-pharmacological intervention?}

In my opinion, non-pharmacological interventions for persons with dementia are those that directly address the unmet needs of this population, unmet needs which result from dementia related symptoms, including memory difficulties, limitations in activities of daily living, or inadequate communication abilities. Unmet needs have been found to provide the most convincing source for behavior problems, and responding to these needs results in a decrease of such behaviors (CohenMansfield, 2013). Given that the most common needs pertain to loneliness, boredom, pain, and discomfort (Cohen-Mansfield et al., 2015), nonpharmacological interventions span a very wide range of actual interventions, of which the papers here mention visual arts (Windle et al., 2017), music (Dyer et al., 2017), exercise programs (Dyer et al., 2017; Morrin et al., 2017), or aromatherapy (Dyer et al., 2017).

But, what about analgesic medication to address pain (Dyer et al., 2017)? Should this intervention be considered non-pharmacological - based on the definition above - or should it be considered pharmacological - as it is in common usage? Furthermore, Morrin et al. (2017) includes deep brain stimulation, electroconvulsive therapy, and repetitive transcranial magnetic stimulation as nonpharmacological interventions. Although these interventions are not pharmacologic, they do not fit the above-suggested definition. Many have lamented the term "nonpharmacological" as it defines a phenomenon by what it is not, and the confusion illustrated above only highlights the need for a clearer definition and better terminology. Would the designation, "need-addressing interventions" be helpful? The term "psychosocial interventions" has been used by some, but it does not seem to capture the full range of treatments. Often addressing the specific need is most useful, such as "engagement" (interventions that address boredom) or "social" interventions (addressing loneliness), but a more global term is needed as well.

It is often difficult to gauge the nature of nonpharmacological interventions from the literature. For example, what is included in "music therapy" (Dyer et al., 2017)? Does it include listening to music via headphones? Is the music individualized to the person's past or current preferences? Is it a group activity of music appreciation? Does it involve singing, using musical instruments, or even dancing? Does the music therapist interact with each participant? It is likely that these different protocols may have a differing impact for different individuals. The paper on visual arts (Windle et al., 2017) provides a very helpful, detailed description of the intervention available on the internet, while the study protocol for persons with young onset 
dementia (van Duinen-van den IJssel et al., 2017) provides only a general outline of the intervention, and will hopefully present a more detailed protocol of the intervention after the study.

In addition to understanding the nature of the intervention, it is important to recognize the elements that make it effective, its "active ingredients." Regrettably, this is often absent from discussions of most non-pharmacological interventions. For example, in the various manifestations of "music therapy," social contact could be an important ingredient, or this may be absent, and sensory stimulation might serve as the active ingredient, or perhaps it is an active connection to past emotions and identity that is the crucial element. Understanding the ingredients will dictate both choice of treatment in specific situations and methods for enhancing the intervention. The paper on visual arts (Windle et al., 2017) does refer to active ingredients, discussing mentally stimulating activities, promotion of error-free positive experiences with opportunities for success, activities without stigma, and the fostering of creative aging. Social participation was included as part of the intervention, and "social inclusion" was a component in that artists were brought into the segregated living area of persons with dementia. Reflection on the active ingredients of intervention is likely to further the development of alternative interventions, to improve current interventions, and to promote the tailoring of interventions to specific needs.

\section{Research concerning subgroups of persons with dementia}

Two of the papers focused on subtypes of dementia, namely Lewy body dementia (Morrin et al., 2017) and young onset dementia (van Duinen-van den IJssel et al., 2017). Lewy body dementia is estimated to comprise between $4.2 \%$ and $7.5 \%$ of all dementia cases, increasing to between $7 \%$ and $11 \%$ when including Parkinson's disease dementia. Young onset dementia is estimated to comprise between $6 \%$ and $9 \%$ of all dementia cases. Thus, it is not surprising that most studies of nonpharmacological interventions for persons with Lewy body dementia are case reports. In fact, this raises a number of underlying questions: Do behavioral problems manifested by persons with specific subtypes of dementia differ from those of other subtypes in their expression or in the circumstances of their appearance? Do persons with Lewy body dementia or with young onset dementia have needs which differ from those of people with other types of dementia? Is there reason to think that non-pharmacological interventions addressing the needs of people with other types of dementia would not be appropriate for people with these subtypes of dementia? Should the principles of intervention be different? And practically, is it realistic to conduct intervention studies for lowfrequency subpopulations?

Obviously, we are aware of differences in the manifestations of different subtypes of dementia, in that, for example, persons with Lewy body dementia exhibit greater fluctuations in function as well as an increased incidence of hallucinations. Yet, since most non-pharmacological interventions are person-centered and tailored to the needs and abilities of the individual, it seems reasonable to incorporate customary non-pharmacological interventions into the care of those manifesting less common types of dementia. If, however, focus on one of the less frequent subtypes is considered to be warranted, this will require large and well-funded studies. Alternatively, with technology, it may be possible to design studies which utilize a network of providers, each of whom could apply the study protocol within his/her professional environment.

\section{Appropriate methodologies}

The multiple methodological issues faced by studies of non-pharmacological interventions have been discussed elsewhere (Cohen-Mansfield et al., 2014). The study of a visual arts program (Windle et al., 2017) is a good example of a convincing mixed methods, multiple measures, multisite study. It is important that real-life clinical interventions be included in our literature and reviews, as their inclusion is more likely to lead to large implementation programs. The inclusion of all types of designs is also a strength of Morrin et al. (2017)'s review. In contrast, reviews that include only RCTs limit the understanding of the available information concerning nonpharmacological interventions.

There is a great need for research on nonpharmacological interventions for persons with dementia. We need to expand the toolkit of interventions, to clarify how to optimize the match between person, behavioral symptom, need, and intervention, and to find ways to promote actual implementation of research findings into different residential settings. In order to move this agenda forward, researchers need to demonstrate more flexibility as well as more rigor. Multiple types of research designs may maximize clinical opportunities. Thus, researchers need to optimize research designs to specific settings, interventions, and budgets. At present, non-pharmacological 
interventions are like "orphan drugs" in that there are no organizations with a commercial incentive to invest large sums in testing them. Therefore, we need to use all opportunities to examine new approaches. Furthermore, the researcher needs to exercise much thoughtfulness in planning the study. Often the label of an assessment does not convey its full content or limitations; different assessments may be more or less appropriate for different stages of dementia; and different methods of administering an intervention may maximize its benefit for persons at different stages of dementia or from different cultural backgrounds.

The basic study of non-pharmacological interventions for persons with dementia is still in its early stages. We therefore look to our authors and the readership to pursue studies that will use this opportunity to enhance the quality of life of persons with dementia and their caregivers.

\section{JISKA COHEN-MANSFIELD}

Minerva Center for the Interdisciplinary Study of End of Life, Tel-Aviv University, Tel Aviv, Israel Department of Health Promotion, School of Public Health, Sackler Faculty of Medicine, Tel-Aviv University, Tel Aviv, Israel

The Herczeg Institute on Aging, Tel-Aviv University, Tel Aviv, Israel Igor Orenstein Chair for the Study of Geriatrics, Tel-Aviv University, Tel-Aviv, Israel

\section{References}

Cohen-Mansfield, J. (2013). Nonpharmacologic treatment of behavioral disorders in dementia. Current Treatment Options in Neurology, 15, 765-785.

Cohen-Mansfield, J., Buckwalter, K., Beattie, E., Rose, K., Neville, C. and Kolanowski, A. (2014). Expanded review criteria: the case of nonpharmacological interventions in dementia. Fournal of Alzheimer's Disease, $41,15-28$.

Cohen-Mansfield, J., Dakheel-Ali, M., Marx, M. S., Thein, K. and Regier, N. G. (2015). Which unmet needs contribute to behavior problems in persons with advanced dementia? Psychiatry Research, 228, 59-64.

Dyer, S. M., Harrison, S. L., Laver, K., Whitehead, C. and Crotty, M. (2017). An overview of systematic reviews of pharmacological and non-pharmacological interventions for the treatment of behavioral and psychological symptoms of dementia. International Psychogeriatrics, 30, 295-309.

Morrin, H., Fang, T., Servant, D., Aarsland, D. and Rajkumar, A. P. (2017). Systematic review of the efficacy of non-pharmacological interventions in people with Lewy body dementia. International Psychogeriatrics, 30, 395-407.

van Duinen-van den IJssel, J. C. L. et al. (2017). Behavior and evolution of young onset dementia part 2 (BEYOND-II) study: an intervention study aimed at improvement in the management of neuropsychiatric symptoms in institutionalized people with young onset dementia. International Psychogeriatrics, 30, 437-446.

Windle, G. et al. (2017). The impact of a visual arts program on quality of life, communication, and well-being of people living with dementia: a mixed-methods longitudinal investigation. International Psychogeriatrics, 30, 409-423. 